\title{
Phenomenology of glider collisions in cellular automaton Rule 54 and associated logical gates*
}

\author{
Genaro Juárez Martínez ${ }^{1}$, Andrew Adamatzky² and \\ Harold V. McIntosh ${ }^{3}$
}

April 2005

${ }^{1}$ Departamento de Programación y Desarrollo de Sistemas, Escuela Superior de Cómputo, Instituto Politécnico Nacional, México DF. E-mail: genarojm@correo.unam.mx

${ }^{2}$ Faculty of Computing, Engineering and Mathematical Sciences, University of the West of England, Bristol, United Kingdom. E-mail:

andrew . adamatzky@uwe. ac.uk

3 Departamento de Aplicación de Microcomputadoras, Instituto de Ciencias, Universidad Autónoma de Puebla, Puebla, México. E-mail:

mcintosh@servidor.unam.mx

\begin{abstract}
Rule 54, a two state, three neighbor cellular automaton in Wolfram's systems of nomenclature, is less complex that Rule 110, but nevertheless possess a rich and complex dynamics. We provide a systematic and exhaustive analysis of glider behavior and interactions, including a cata$\log$ of collisions. Many of them shows promise are computational elements.

Keywords: cellular automata, gliders, collision-based computing
\end{abstract}

\section{Introduction}

We study one-dimensional (1D) binary state ternary neighborhood cellular automata $(\mathrm{CA})$ exhibiting a sophisticated space-time dynamics of traveling localized patterns, and deal with cell state transition Rule 54, mainly due to its ability to generate rich varieties of periodic structures, known as particles or gliders [10]. Rule 54 belongs to a class of complex CA rules - Rule 54 is an example of Wolfram's class IV rules [15] — which includes The Game of Life [4], High Life [3], Life-3d [6], Rule 54 [5], Rule 110 [7] and Beehive Rule [17]. These CA produce gliders in their evolution, and thus pose a fruitful subject of investigations not only in terms of complex systems and self-organization

\footnotetext{
* Journal Chaos, Fractals and Solitons, by publish, 2005
} 


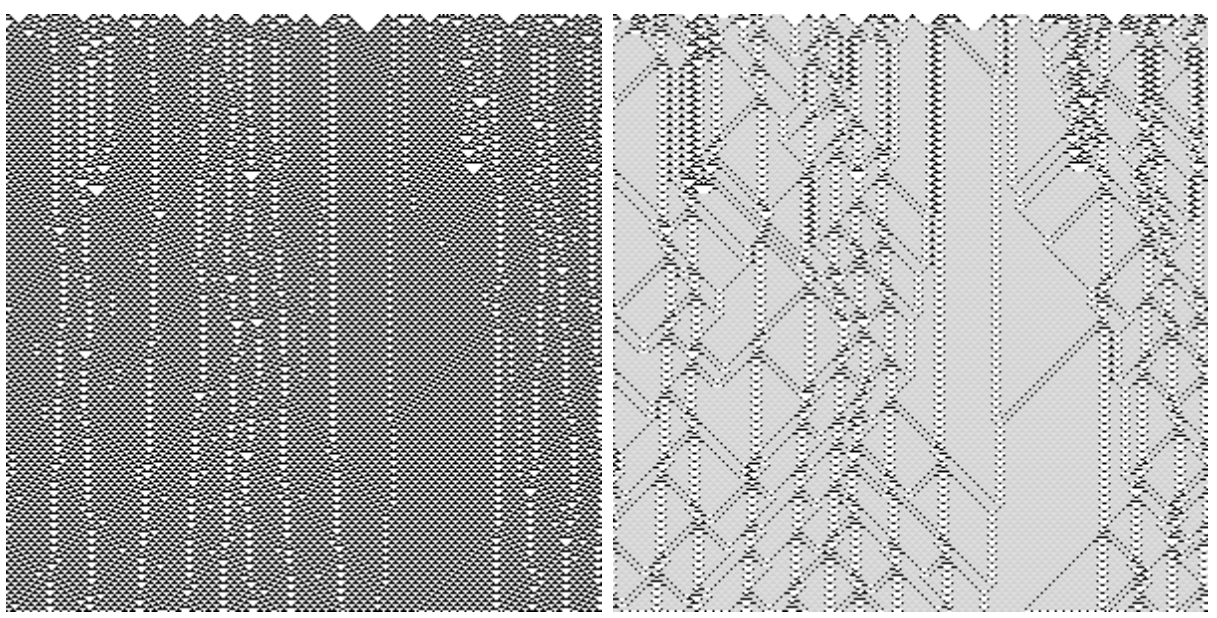

Figure 1: Examples 1D CA governed by Rule 54, random initial configuration. Time goes down.

but also as novel substrate for dynamics non-classical computation [1]; see also universality of Rule $110[7,16]$.

We study $1 \mathrm{D} C A-1 \mathrm{D}$ array of cells, indexed by integer $i$ - where all cells take states from set $\{0,1\}$, and update their states in parallel in discrete time depending on their current states and states of their closest (left and right) neighbors according to cell state transition function $\varphi: x_{i}^{t+1}=\varphi\left(x_{i-1}^{t}, x_{i}^{t}, x_{i+1}^{t}\right)$. The Rule 54 studied in the paper has the following explicit state transition table (sequence 01101100 is 54 in decimal notation, so name of the rule):

\begin{tabular}{ccc|c}
$x_{i-1}^{t}$ & $x_{i}^{t}$ & $x_{i+1}^{t}$ & $x_{i}^{t+1}$ \\
\hline 0 & 0 & 0 & 0 \\
0 & 0 & 1 & 1 \\
0 & 1 & 0 & 1 \\
0 & 1 & 1 & 0 \\
1 & 0 & 0 & 1 \\
1 & 0 & 1 & 1 \\
1 & 1 & 0 & 0 \\
1 & 1 & 1 & 0
\end{tabular}

An example of space-time configurations of 1D CA with Rule 54 is shown in Fig. 1, where white pixels are cells in state 0 , and black pixels are cells in state 1. The second picture show a filter for see best ether (periodic background) and gliders.

Fig. 2 illustrate behavior of the automaton which initial configuration has $0^{*} 10^{*}$ a single cell in state 1 , rest of the cells are in state 0 . This may indicate they Rule 54 is not so 'complex' as say Rule 110, because CA governed by Rule 110 and developing from initial configuration $0^{*} 10^{*}$ does not reach a quasi-stable 


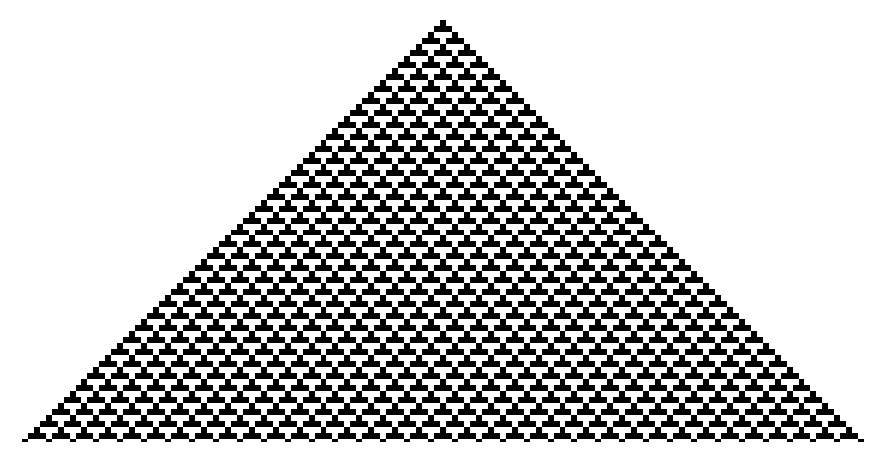

Figure 2: Development from initial configuration $0^{*} 10^{*}$. Time goes down.

orbit until approximately 3,400 time steps.

In their pioneer work Boccara, Nasser and Roger [5] produce a list of gliders known at that time, ${ }^{1}$ and discuss existence of glider gun; they also applied some statistical analysis to analyzing stability of gliders. Later Hanson and Crutchfield [8] introduced a concept of "computational mechanics" - applied finite state machine language representation in studying defect dynamics in $1 \mathrm{D}$ $\mathrm{CA}$, and in deriving motion equations of filtered gliders. More studies were undertaken by Wolfram [16], who exhibit glider collisions with long period of after-development and several filters for detecting gliders and defects, and Martin [11], who designed an algebraic group of order four to represent collisions between basic gliders. In present paper we advance these results in several direction: design a single filter to detect all gliders, discover novel glider guns, demonstrate that all gliders in Rule $54 \mathrm{CA}$ can be produced via collisions of gliders, and produce a catalog of all outcomes of all possible collisions ${ }^{2}$ between two and three gliders. We also apply these findings to construct dynamical logical gates, where Boolean values are represented by the gliders.

\section{Gliders in Rule 54 CA}

We may specify two types of glider - natural, formed without 'help' of internal collisions or any other kinds of compositions of gliders - and - compound, produced by internal collisions of natural gliders. Fig. 3 illustrates previously known gliders: $w^{\rightarrow}, w^{\leftarrow}, g_{o}$ and $g_{e}$ natural gliders, and glider gun is a compound glider. ${ }^{3}$

Properties of gliders, and also ether $e_{1}$ and $e_{2},{ }^{4}$ are listed in Table 1, where

\footnotetext{
${ }^{1}$ In present paper we adopt classification of gliders developed in [5]

${ }^{2}$ We used OSXLCAU21 system for OPENSTEP, Mac OS X and Windows to visualize glider collisions; this can be download of http://www.rule110.org/download/

${ }^{3}$ The existence of glider gun in Rule $54 \mathrm{CA}$ demonstrates unlimited growth of configurations in Rule $54 \mathrm{CA}$

${ }^{4}$ Ether $e_{1}=(1000)^{*}$ and shift $e_{2}=(1110)^{*}$
} 

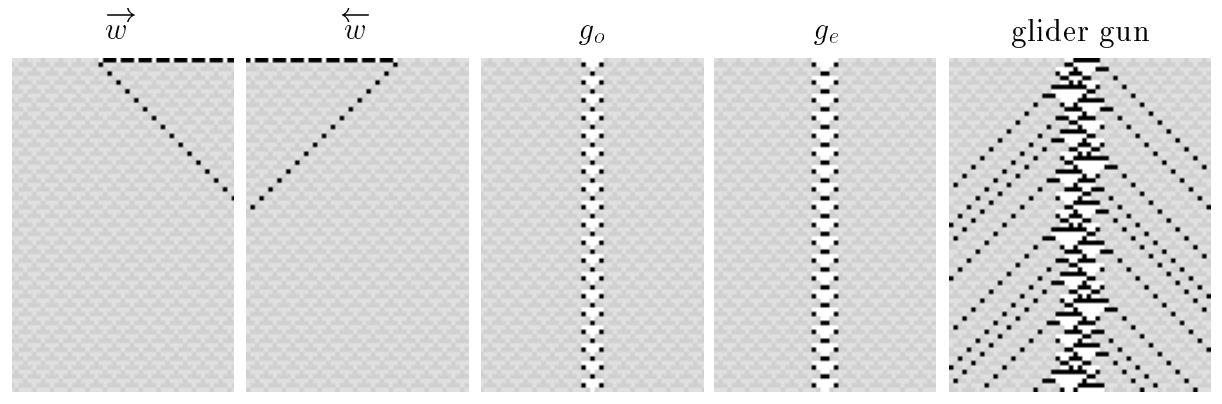

Figure 3: Space-time configuration of Rule $54 \mathrm{CA}$ with basic, previously known, gliders in Rule $54 \mathrm{CA}$.

second column shows velocity $v_{g}{ }^{5}$ third column indicates maximal and minimal sizes of gliders (there are the same if glider does not breath), last column indicates if glider can cover the whole space (' $\mathrm{T}$ ' means total covering, ' $\mathrm{P}$ ' partial covering).

\begin{tabular}{||c|c|c|c||}
\hline \hline structure & $v_{g}$ & width & cap \\
\hline \hline$e_{1}$ & $2 / 2=1$ & 4 & $\mathrm{~T}$ \\
\hline$e_{2}$ & $2 / 2=1$ & 4 & $\mathrm{~T}$ \\
\hline$w^{\rightarrow}$ & $2 / 2=1$ & 2 & $\mathrm{P}$ \\
\hline$w^{\leftarrow}$ & $-2 / 2=-1$ & $0-4$ & $\mathrm{P}$ \\
\hline$g_{o}$ & $0 / 4=0$ & $6-2$ & $\mathrm{~T}$ \\
\hline$g_{e}$ & $0 / 4=0$ & $7-3$ & $\mathrm{~T}$ \\
\hline glider gun & $0 / 32=0$ & $14-4$ & $\mathrm{P}$ \\
\hline \hline
\end{tabular}

Table 1: Characterization of gliders in Rule 54.

Fig. 4 show an interaction of gliders streams with stationary localizations, the localization are transformed to glider guns in result of these interactions, some gliders are destroyed during interaction (this means that result frequency of glider streams can be controlled by stationary localizations) (Fig. 4a). In Fig. 4 b we see another type of glider gun 'extension' composed by two $g_{e}$ gliders.

\section{Collisions between glider in Rule 54 CA}

We uncover phenomenology of glider collision in a systematic way. 


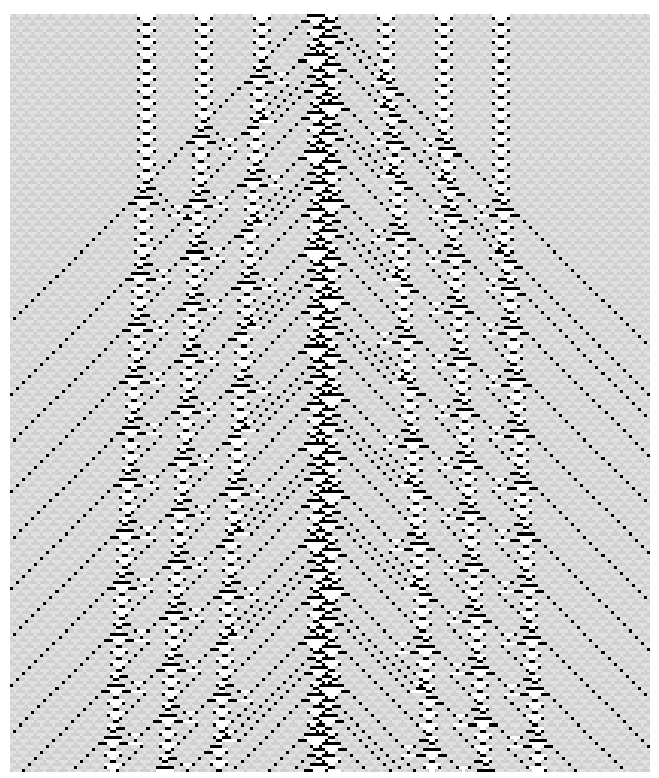

a
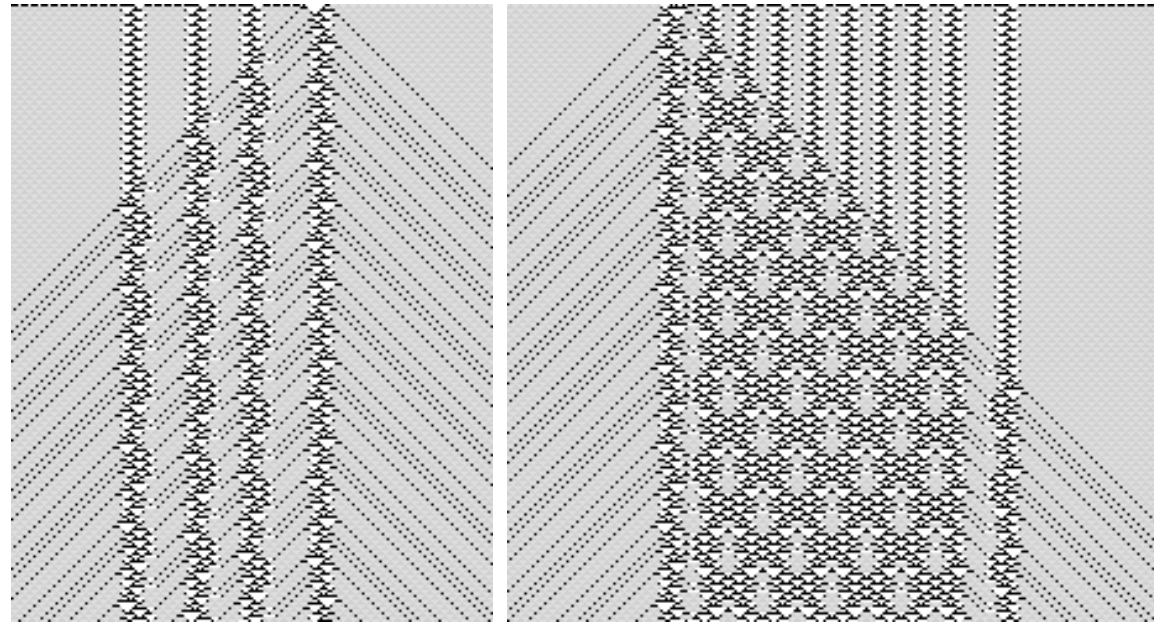

$\mathrm{b}$

Figure 4: Interaction of glider streams with stationary localizations produce novel glider guns. 


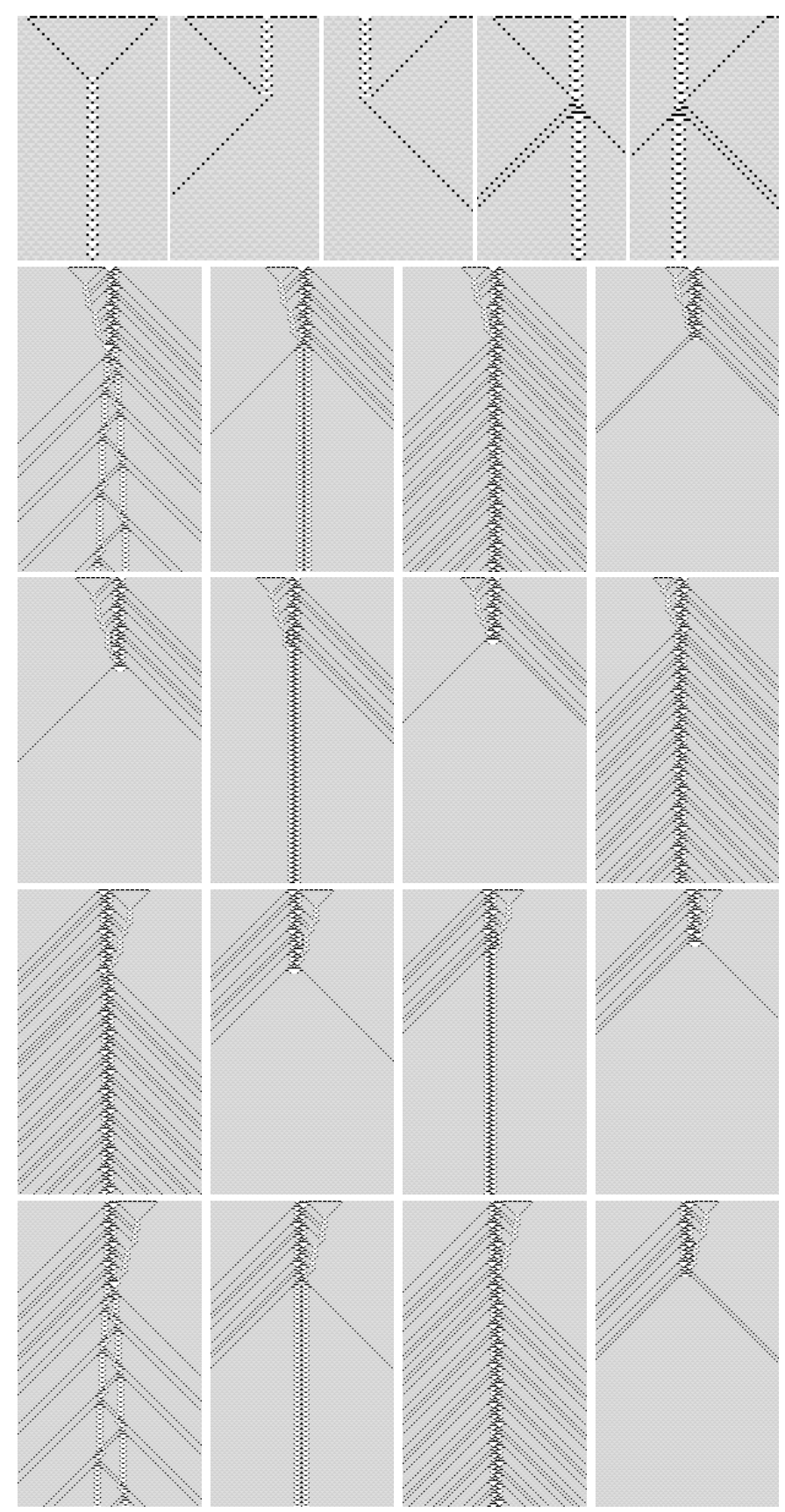

Figure 5: Space-time configurations of Rule 54 CA illustrating binary collisions between gliders. 


\subsection{Binary collisions}

Fig. 5 illustrates all possible types of binary collisions between gliders in Rule 54 CA. Collisions between $w^{\rightarrow}, w^{\leftarrow}, g_{o}$ and $g_{e}$ gliders were already known before. However collisions between $w^{\rightarrow}$ and $w^{\leftarrow}$ gliders against glider gun are novel. Essentially, we only played with initial distances between glider w's and glider gun to obtain all types binary collisions. Counting configurations in Fig. 5 from left to the right and top down we observe the following types of collisions:

- fusion of two gliders in a stationary localization (1st configuration),

- reflection of a glider by stationary localization with annihilation of the localization (2nd and 3rd configurations),

- multiplication and reflection of glider collided with stationary localization (4th and 5th configurations),

- formation of a quasi glider gun during collision of a glider to glider gun (6th and 18th configurations),

- transformation of glider gun to a stationary localization by glider colliding to a gun (7th, 11th, 16th and 19th configurations),

- self-repair of glider gun partially damaged in result glider collision (8th, 13th, 14th, 19th and 20th configurations). Gliders across as soliton [14].

\subsection{Triple collisions}

Fig. 6 illustrate all triple collisions between natural gliders. We omitted collisions with glider gun because are just variants of binary collisions, discussed before.

The following types of collision outcomes (Fig. 6) could be highlighted:

- annihilation of gliders and stationary localization (1st configuration),

- gliders pass through stationary localization and neither of patterns involved in collision destroyed (2nd configuration) as soliton,

- gliders are reflected or delayed by stationary localization and one of the gliders is multiplied (e.g. 3rd configuration); the stationary localization shifted to few sites left or right (usually in direction opposite to that where multiplied gliders travel), or transformed to another type of stationary localization (e.g. 8th configuration),

- colliding of a glider train of two gliders with a stationary localization may cause annihilation of gliders and spatial shift of the stationary localization (e.g 9th and 10th configurations) or reflection of the train and formation of two new gliders (12th and 14th configuration),

\footnotetext{
${ }^{5}$ Velocity is determined dividing displacement between period, where $g$ is a specific glider in Rule $54 \mathrm{CA}$
} 
- collision of a glider to a stationary localization leads to formation of a glider gun (e.g. 29th - 30th configurations) or quasi glider gun (e.g. 27th - 28th configurations).

\subsection{Other collisions}

Colliding glider trains with clusters of stationary localizations lead to an impressive outcomes, as shown in Fig. 7:

- when train of three $w^{\rightarrow}$ gliders collide to a cluster of five localizations $g_{e}$, two gliders are annihilated and the one on glider is reflected, glider $w$ 's are generated, while the cluster of localizations $g_{e}$ remains intact (1st configuration in Fig. 7),

- train of two gliders collided to cluster of three localizations $g_{e}$ is reflected and distance between localization in the cluster decreases (2nd configuration), compressing of stationary domains by gliders,

- all other collisions between glider trains and stationary clusters lead to increase of the cluster diameter with decrease of number of localizations in the cluster, pruning by gliders, and either reduction (4th and 5th configurations) or multiplication (6th and 7th configurations) of glider streams.

Space-time dynamics of CA undergoes a transient period of perturbations after the collision. In most case this transient period is pretty short, however collision shown in 7 th configuration in Fig. 7 leads to a transient period of at least 380 time steps.

Fig. 8 show that so-called black holes and glider eaters exist in development of Rule $54 \mathrm{CA}$. Two black hole are shown in Fig. 8: tiles $T_{8}$ localization absorbs, or annihilate, gliders $w^{\rightarrow}$ and $w^{\leftarrow}$ (Fig. 8a) and stationary localization $g_{0}$ absorbs pairs of gliders $w^{\rightarrow}$ and $w^{\leftarrow}$ (Fig. 8b). In Fig. $8 \mathrm{c}$ we can see glider $w^{\rightarrow}$ eating pairs of gliders $w^{\leftarrow}$ while traveling to the right.

\subsection{Producing gliders by collisions}

Are all types gliders produced via collisions? Boccara, Nasser and Roger [5] established that gliders $w^{\rightarrow}, w^{\leftarrow}$ and $g_{o}$, and glider gun can be constructed through a collision; this was also verified by our experiments. Also we demonstrated that a glider gun can be produced in a triple collisions of gliders.

We can also produce gliders in other types of collisions, e.g. in situations when initial configuration has a domain of 0 's and gliders are traveling boundaries of the domain. Thus Fig. 9 shows that glider $g_{e}$ is a product of collision between gliders $w^{\rightarrow}$ and $w^{\leftarrow}$. By utilizing various types of ether in initial conditions we can produce all types of natural gliders.

Fig. 10 illustrates that a novel glider gun besides extended, its produced in a collision between one defect (glider) and three stationary localizations.

Table 2 describes series of glider collisions required to produce basic types of gliders and glider guns. 


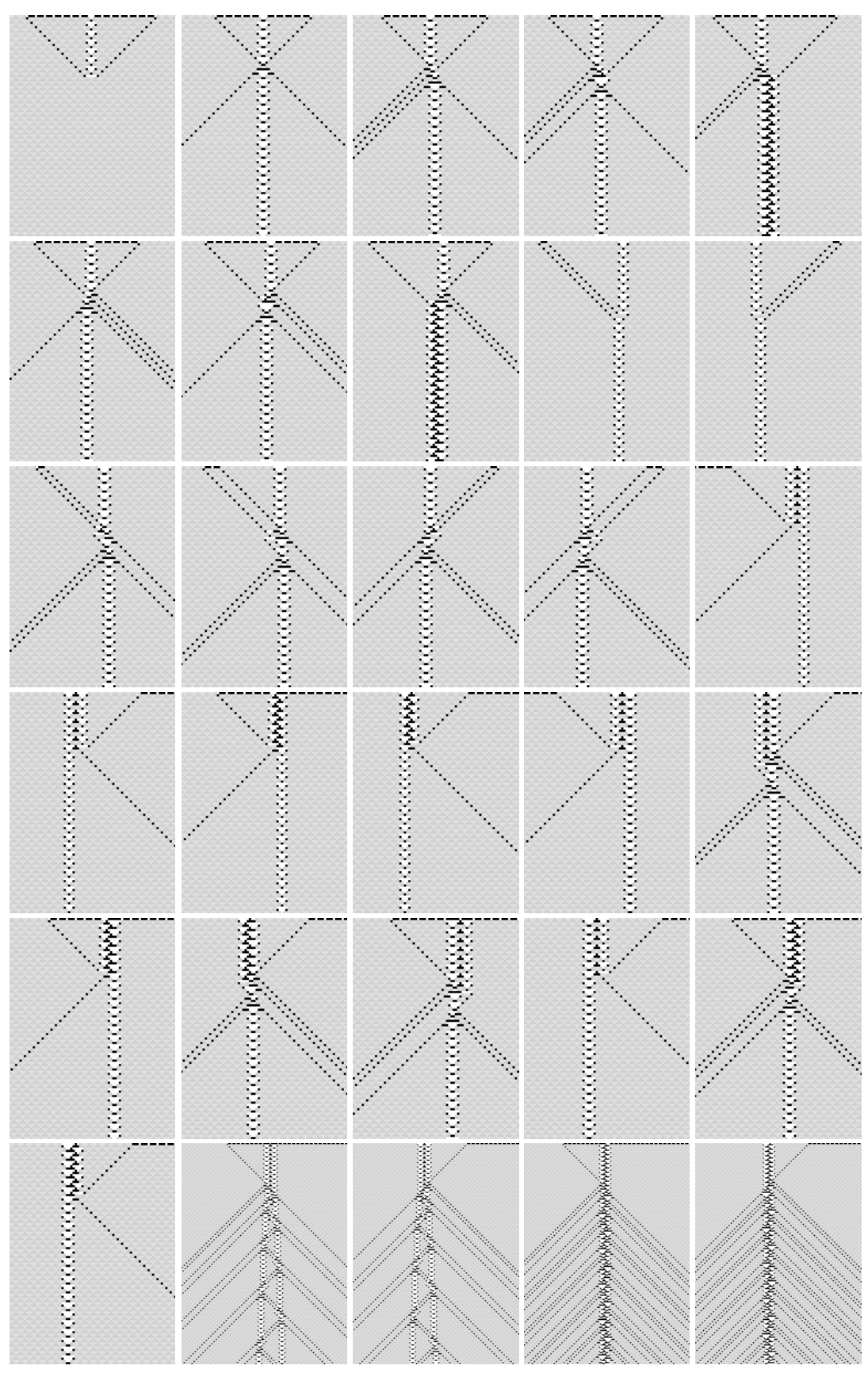

Figure 6: Space-time configurations of Rule $54 \mathrm{CA}$ illustrating ternary glider collisions. 


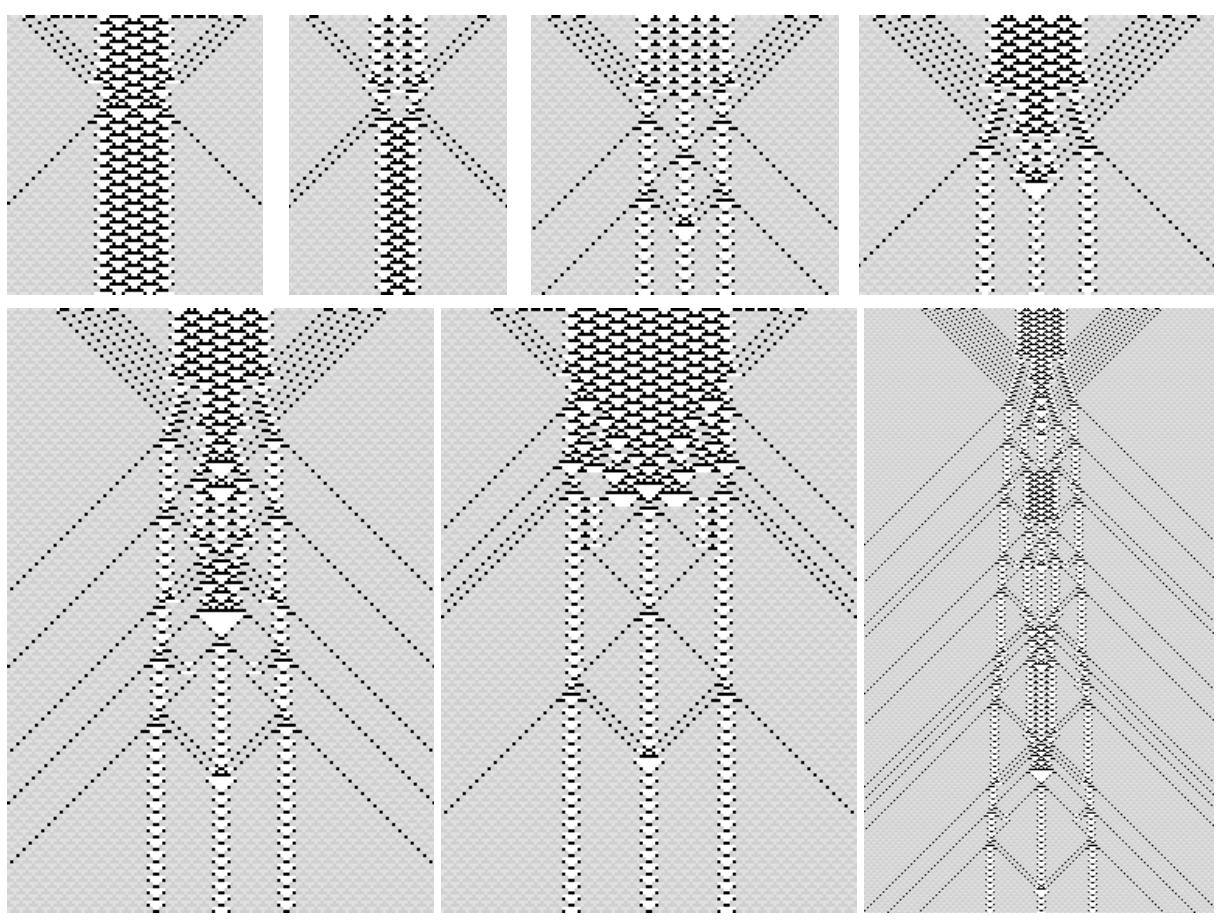

Figure 7: Space-time configuration of 1D CA demonstrating outcomes of colliding glider trains with clusters of stationary localizations. Time goes down.

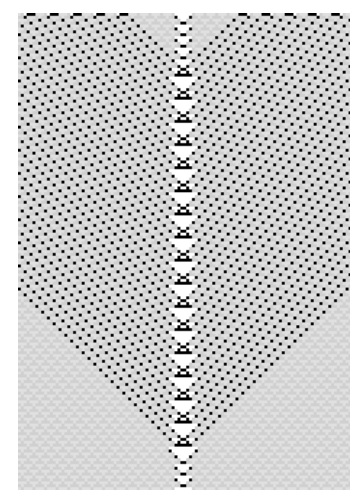

(a)

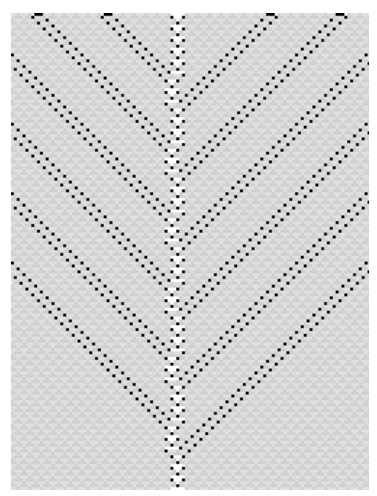

(b)

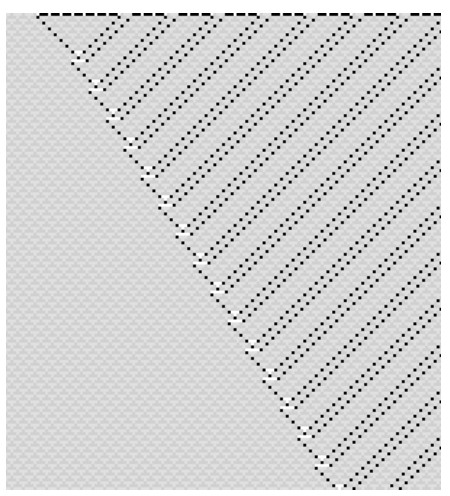

(c)

Figure 8: Space-time configurations of 1D CA demonstrating existence of black holes (a) and (b), and glider eater (c). Time goes down. 


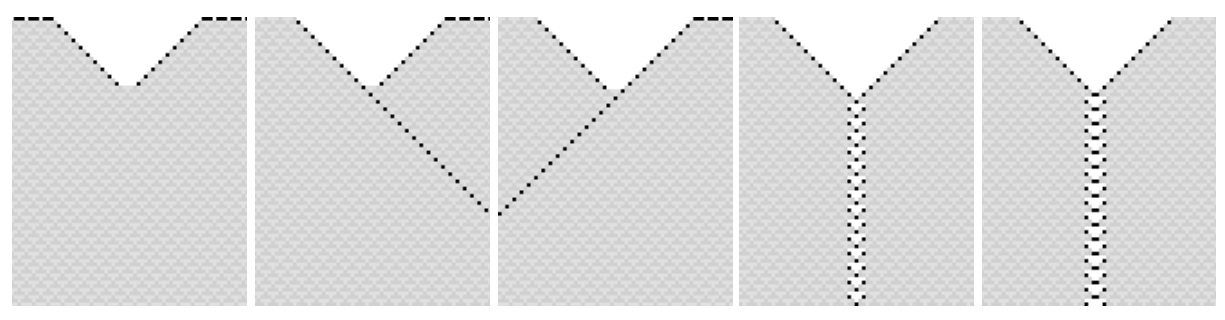

Figure 9: Space-time configuration of 1D CA demonstrating production of natural gliders by traveling boundaries of domain of 0 's. Time goes down.
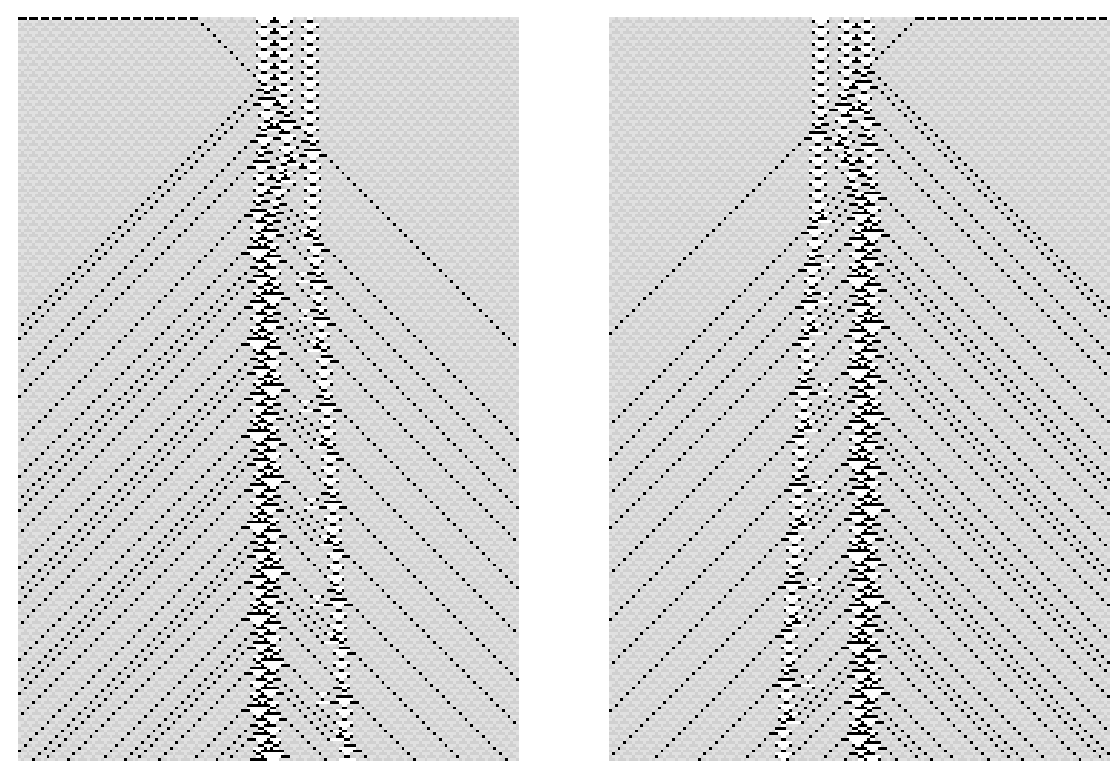

Figure 10: Space-time configuration of 1D CA demonstrating production of a novel glider gun besides extended in Rule $54 \mathrm{CA}$. Time goes down.

\begin{tabular}{||c|l||}
\hline \hline glider & production sequence \\
\hline \hline$w^{\rightarrow}$ & $g_{o}, w^{\leftarrow} ; e_{1}{ }^{*} 0^{4 n-2} e_{2}{ }^{*} \forall n>0$ \\
\hline$w^{\leftarrow}$ & $w^{\rightarrow}, g_{o} ; e_{1}{ }^{*} 0^{4 n} e_{2}{ }^{*} \forall n>0$ \\
\hline$g_{o}$ & $w^{\rightarrow}, w^{\leftarrow} ; e_{1}{ }^{*} 10^{n} e_{1} * \forall n>0$ and odd \\
\hline$g_{e}$ & $e_{1}{ }^{*} 10^{n} e_{1}{ }^{*} \forall n>0$ and even \\
\hline glider gun & $w^{\rightarrow}, 2 g_{e} ; 2 g_{e}, w^{\leftarrow}$ \\
\hline glider gun $^{n}$ & $w^{\rightarrow}, g_{e}, 2 g_{e} ; 2 g_{e}, g_{e}, w^{\leftarrow}$ \\
\hline \hline
\end{tabular}

Table 2: Collision sequence for glider production. 


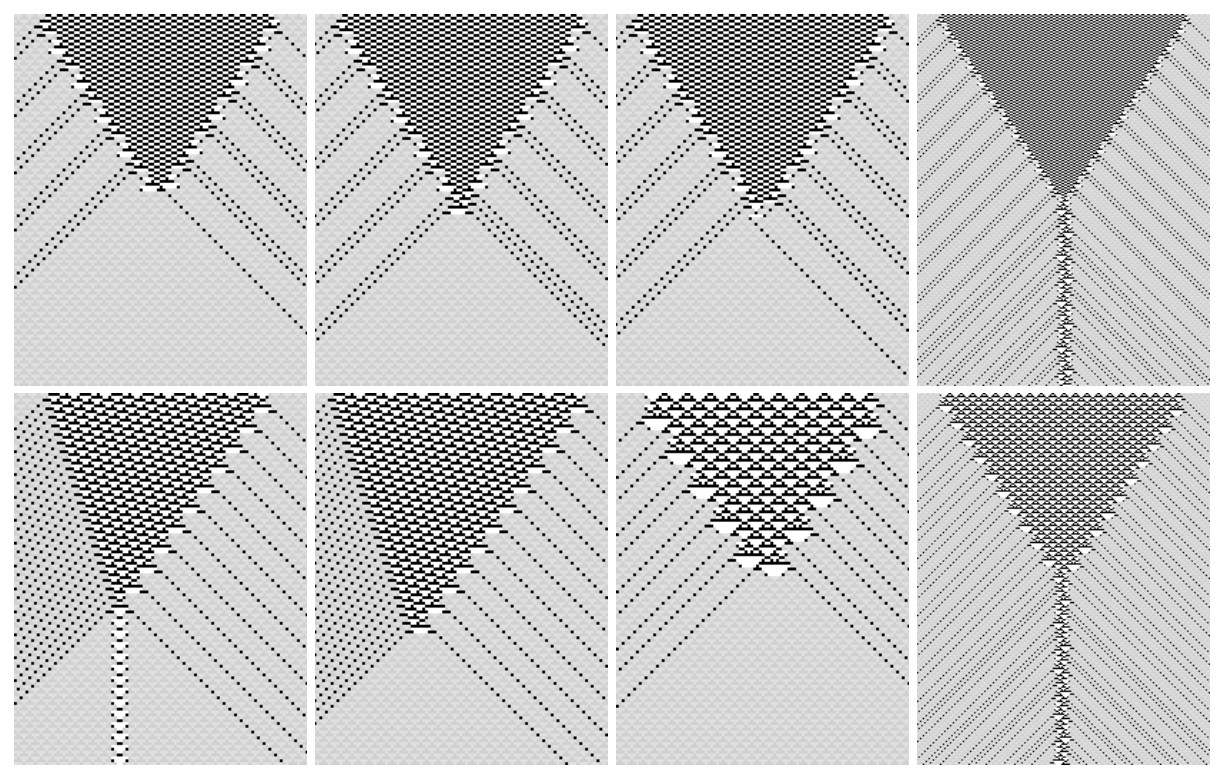

Figure 11: Erosions

\subsection{Development of tiles}

A finite domain of tiles always decrease in development of 1D CA Rule 54 configurations (Fig. 9), however during this erosion gliders and glider guns are produced. As shown in Fig. 9 common types of tiles - in initial conditions of finite tile domains - develop in several ways:

- domain boundaries emit several duplets of gliders during annihilation (1st, 2nd, 3rd, 6th, 7th configurations),

- domain converges to a stationary localization (5th configuration),

- domain converges into a glider gun (4th and 8th configurations).

\section{Computing with gliders in Rule $54 \mathrm{CA}$}

A minimal gate $\langle x, y\rangle \rightarrow\langle x$ AND $\operatorname{NOT}(y), \operatorname{NOT}(x)$ AND $y\rangle$ (Fig. 12a) is realized when two gliders $w^{\rightarrow}$ and $w^{\leftarrow}$ in the same phase collide and annihilate in the result of collision (Fig. 9, 1st configuration). If presence of $w^{\rightarrow}$ and $w^{\leftarrow}$ gliders corresponds to TRUE value of logical variables $x$ and $y$, then undisturbed trajectories of the gliders gives us values of operation $x$ AND NOT $(y)$ and operation $\operatorname{NOT}(x)$ AND $y$, respectively.

When colliding $w^{\rightarrow}$ and $w^{\leftarrow}$ gliders are in anti-phase, see Fig. 5, 1st configuration, a stationary breather $g_{o}$ is formed. Encoding logical variables as 


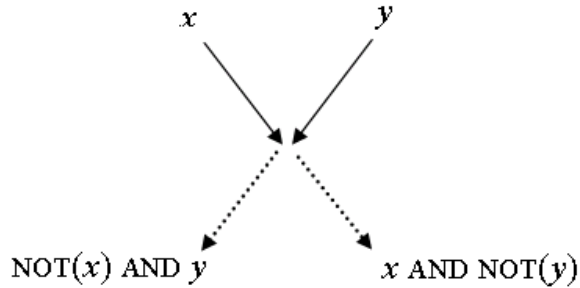

(a)

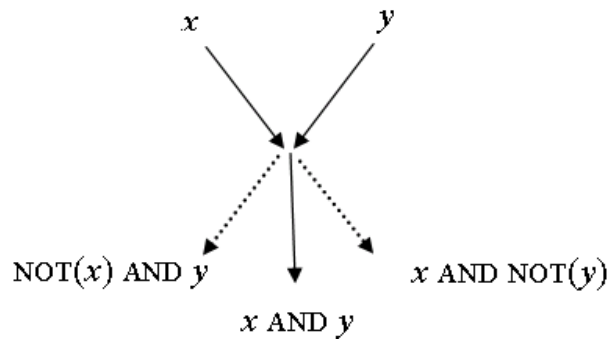

(b)

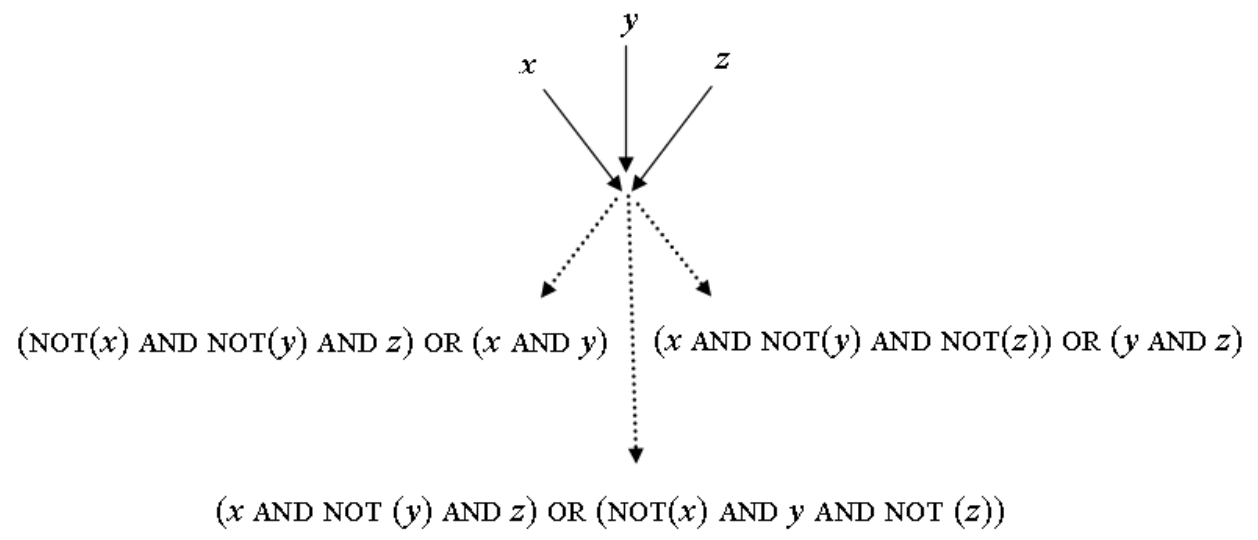

(d)

Figure 12: Elementary gates implemented by colliding gliders and breathers in Rule 54 1D CA. 
previously, we see $g_{o}$ represents value of conjuction $x$ AND $y$, and thus gate is implemented $\langle x, y\rangle \rightarrow\langle\operatorname{NOT}(x)$ AND $y, x$ AND $y, x$ AND NOT $(y)\rangle$ with two inputs and three outputs (Fig. 12b).

The stationary localization $g_{o}$ can be used further to construct other varieties of gate. Thus, when glider $w^{\rightarrow}$ collides to $g_{o}$ (Fig. 5, 2nd configuration), breather $g_{o}$ collapses and the glider is reflect (transformed to $w^{\leftarrow}$ ). If $w^{\rightarrow}$ represents $x$ and $g_{o}-y$ then their undisturbed trajectories (dotted lines in Fig. 12c) give us values of operation NOT $x$ AND $y$ and operation $x$ AND NOT $y$, while trajectory of newly formed $w^{\leftarrow}$ represents $x$ AND $y$. These represent dynamical architecture of gate $\langle x, y\rangle \rightarrow\langle x$ AND $y, \operatorname{NOT}(x)$ AND $y, x$ AND NOT $(y)\rangle$ (Fig. 12c).

All localizations annihilate when gliders $w^{\rightarrow}$ (representing value of $x$ ) and $w^{\leftarrow}$ (value of $z$ ) collide to breather $g_{o}$ (value of $y$ ) (Fig. 6, 1st configuration). If after collision glider $w^{\rightarrow}$ emerges along it is (seems to be undisturbed) trajectory this means that either only $w^{\rightarrow}$ was present before collision or $w^{\rightarrow}$ was absent but glider $w^{\leftarrow}$ collided to $g_{o}$; the same applies to undisturbed trajectory of glider $w^{\leftarrow}$ (this due to collision shown in Fig. 5, 2nd configuration. We shown early that breather $g_{o}$ can be formed in collision of $w^{\rightarrow}$ and $w^{\leftarrow}$, therefore presence of $g_{o}$ after collision may indicate either both or none gliders were present in the collision. The triple collision realizes three-input three-output gate $\langle x, y, z\rangle \rightarrow\langle(\operatorname{NOT}(x)$ AND $\operatorname{NOT}(y)$ AND $(z))$ OR $(x$ AND $y),(x \operatorname{AND} \operatorname{NOT}(y)$ AND $z)$ OR $(\operatorname{NOT}(x)$ AND $y$ AND $\operatorname{NOT}(z))$, $(x \operatorname{AND} \operatorname{NOT}(y) \operatorname{AND} \operatorname{NOT}(z))$ OR $(y \operatorname{AND} z))$.

Some scenarios of multiple-collisions of localizations allow us to realize operations of reflection, multiplication and delay. For example, as seen in Fig. 5, 4th configuration, when glider $w^{\rightarrow}($ signal $x)$ collides to breather $g_{e}$, it reflects first glider $w^{\leftarrow}$ in Fig. 5, 4th configuration, represents reflection $x_{r}$ of signal $x$ (Fig. 13a). Also in result of the collision two more gliders are produced: second glider $w^{\leftarrow}$ (signal $x_{r d}$ in Fig. 13a) - reflected and delayed signal $x$, and glider $w^{\rightarrow}$ (signal $x_{d}$ in Fig. 13a) - delayed signal $x$. In this signal $x$ is delayed $\left(x_{d}\right)$ and its reflection is multiplied $\left(x_{r}\right.$ and $\left.x_{r d}\right)$.

A distance between gliders in a stream can be increased by colliding the stream with breather $g_{e}$. In Fig.6, 11th configuration, train of two gliders $w \rightarrow$ and $w^{\leftarrow}$ collides to $g_{e}$, and reflected (reflection of signals $x$ and $y$ to $x_{r}$ and $y_{r}$ in Fig. 13b). At the same time, two more $w^{\rightarrow}$ gliders, the first newly born $w^{\rightarrow}$ can be interpreted as signal $x$ which passed the collision undisturbed and the second $w^{\rightarrow}$ (signal $y_{d}$ in Fig. 13b) as delayed signal $y$. Interval between signals $x$ and $y$ was increased by collision with stationary localization; this can be also interpreted as a selective delay of second signal $y$.

A threshold element can be constructed based on collision of glider $w^{\rightarrow}$ to tuple of breathers $g_{o}$ (Fig. 6, 15th configuration). In this collision, the gliders is reflected and one of the breathers is destroyed (Fig. 13c). Thus, arranging $m$ breather in a row we built a threshold element, which allows only $m+1$ th glider in a stream to pass through. However the element is not reusable.

When tuple of gliders $w^{\rightarrow}$ collides to breather $g_{o}$ (Fig. 6, 9th configuration) both gliders annihilate but at time of annihilation they "pull up" $g_{o}$ (Fig. 13d). This can be interpreted as a shift of non-negative integer, represented by $g_{o}$, in 


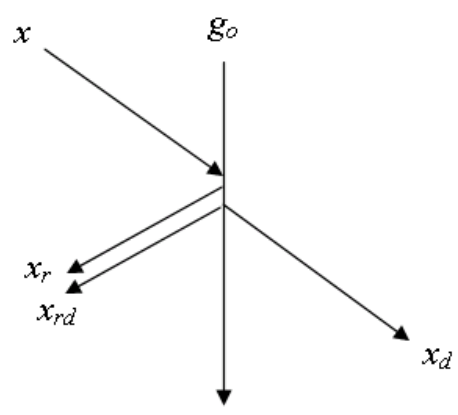

(a)

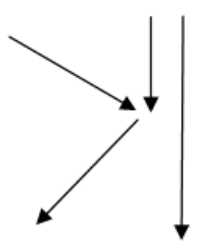

(c)

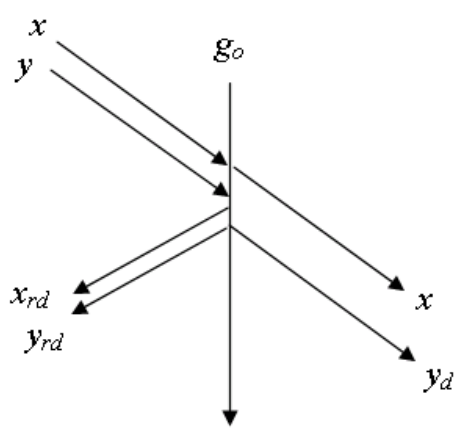

(b)

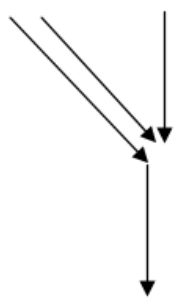

(d)

Figure 13: Operations implemented by colliding gliders and breathers in Rule 54 1D CA: (a) reflection, multiplication and delay; (b) reflection and selective delay of signals; (c) elementary unit of a threshold element; (d) shift operation. 
a storage device.

So far we got enough collision scenarios to say that a counter machine can be simulated in Rule $54 \mathrm{CA}$. We can represent an infinite storage device by pattern of stationary localizations, breathers $g_{o}$; the information can be written in the device (formation of $g_{0}$ in collision of $w^{\rightarrow}$ and $w^{\leftarrow}$, Fig. 5, 1st configuration), erased ( $g_{o}$ is annihilated in collision with $w^{\rightarrow}$ or $w^{\leftarrow}$, Fig. 5, 2nd and 3th configuration), read and shifted (Fig. 6, 6th and 9th configuration); and, check of whether storage is empty can be implemented (Fig. 6, 15th configuration). These operations are enough to simulate a counter machine. The combination of counter machines will simulate a Turing machine, which must demonstrate Turing universality of Rule $54 \mathrm{CA}$.

\section{Conclusions}

We undertook a detailed study of localization dynamics in Rule 54 1D CA. We shown that a complete list of gliders is produced through collisions in two different ways and a two novel glider guns besides extended is also generated via glider collisions. Rich collision dynamics in Rule $54 \mathrm{CA}$ allowed us to construct several kind of computation operations and, in general, computing abilities of Rule $54 \mathrm{CA}$. We envisage the Rule $54 \mathrm{CA}$ will make a good alternative to Rule $110 \mathrm{CA}$ in a sense of logical universality as well as simulation of a Turing machine.

\section{Acknowledgements}

To David Hillman for important commentaries on Rule 54 (Hillman determine the group of order four in Rule 54, although not publish his result). To support of British Council Mexico.

\section{References}

[1] Andrew Adamatzky, Computing in Nonlinear Media and Automata Collectives, Institute of Physics Publishing, Bristol and Philadelphia, 2001. (ISBN 0-7503-0751-X)

[2] Andrew Adamatzky (Ed.), Collision-Based Computing, Springer, 2002. (ISBN 1-85233-540-8)

[3] David I. Bell, "HighLife - An Interesting Variant of Life," http://www.tip.net.au/ dbell/, 1994.

[4] Elwyn R. Berlekamp, John H. Conway and Richard K. Guy, Winning Ways for your Mathematical Plays, Academic Press, 1982 (ISBN 0-12-091152-3) vol. 2, chapter 25 . 
[5] N. Boccara, J. Nasser and M. Roger, "Particle like structures and their interactions in spatio-temporal patterns generated by one-dimensional deterministic cellular automaton rules," Physical Review A 44, No. 2, pp. 866-875, July 1991.

[6] Carter Bays, "Candidates for the Game of Life in Three Dimensions," Complex Systems 1, pp. 373-400, 1987.

[7] Matthew Cook, "Universality in Elementary Cellular Automata," Complex Systems, Volume 15, Number 1, pp. 1-40, 2004.

[8] James E. Hanson and James P. Crutchfield, "Computacional Mechanics of Cellular Automata: An Example," Physics D 103 (1-4), pp. 169-189, 1997.

[9] Genaro Juárez Martínez and Harold V. McIntosh, "ATLAS: Collisions of gliders like phases of ether in Rule 110," http://delta.cs.cinvestav.mx/ ${ }^{\sim}$ mcintosh/comun/s2001/s2001.html, August 2001.

[10] Genaro Juárez Martínez, Harold V. McIntosh and Juan Carlos Seck Tuoh Mora, "Gliders in Rule 110," submitted to International Journal of Unconventional Computing, August 2004.

[11] Bruno Martin, "A Group Interpretation of Particles Generated by OneDimensional Cellular Automaton, Wolfram's Rule 54," International Journal of Modern Physics C, Vol. 11, No. 1, pp 101-123, 2000.

[12] Harold V. McIntosh, "Rule 110 as it relates to the presence of gliders," http://delta.cs.cinvestav.mx/ ${ }^{\sim}$ mcintosh/oldweb/pautomata.html, January 1999.

[13] Melanie Mitchell, Computation in Cellular Automata: A Selected Review, Santa Fe Intitute, NM 87501 U.S.A., September 1996.

[14] James K. Park, Kenneth Steiglitz and William P. Thurston, "Soliton-like behavior in automata," Physica D 19, 423-432, 1986.

[15] Stephen Wolfram, Theory and Aplications of Cellular Automata, World Scientific Press, Singapore, 1986. (ISBN 9971-50-124-4 pbk)

[16] Stephen Wolfram, A New Kind of Science, Wolfram Media, Inc., Champaign, Illinois, 2002. (ISBN 1-57955-008-8)

[17] Andrew Wuensche, "Self-reproduction by glider collisions; the beehive rule," International Journal Pollack et. al, editor, Alife9 Proceedings, pp. 286-291, MIT Press, 2004. 